\title{
An experimental study of thermal diffusivity of Au nanoparticles: effects of concentration particle size
}

\author{
Esmaeil Shahriari $^{1,2} \cdot$ Mohammad Moradi $^{1,2} \cdot$ Morteza Raeisi $^{1}$
}

Received: 10 January 2016/ Accepted: 25 May 2016/Published online: 18 June 2016

(c) The Author(s) 2016. This article is published with open access at Springerlink.com

\begin{abstract}
In this article, Au nanoparticles in polyvinylpyrrolidone (PVP) solution were prepared by gamma radiation at different concentrations. The solutions were irradiated at doses of $50 \mathrm{kGy}$ for making different sizes. The average sizes of particle in the prepared samples were measured using the nanophox machine. A dual-beam mode-mismatched thermal lens (TL) method was used to investigate the effect of thermal diffusivity of samples. The TL measurement was carried out using a green diode laser (wavelength $532 \mathrm{~nm}, 60 \mathrm{~mW}$ ) and a $\mathrm{He}-\mathrm{Ne}$ laser (wavelength $632.8 \mathrm{~nm}, 0.5 \mathrm{~mW}$ ) for excitation source and probe beam, respectively. The results showed that the thermal diffusivity of samples enhances with the growth of particle size and density of solutions. This increment can be attributed to phonon scattering at interface of particlesliquid and contact between the nanoparticles and surrounded liquid.
\end{abstract}

Keywords Au nanoparticles - Thermal lens method · Thermal diffusivity

\section{Introduction}

Nanoparticles, such as metals, are of great interest for a wide variety of applications in electronics [1] and optical components [2], heat transfer fluids in automotive [3],

Esmaeil Shahriari

esmaeil.phy@gmail.com

1 Department of Physics, Faculty of Science, Shahrekord University, Shahrekord, Iran

2 Photonic Research Group, Shahrekord University, Shahrekord, Iran electronic cooling, medical therapies, and drug delivery [4-7] due to their unique or improved properties determined primarily by size, composition, and structure along with their self-organized film structures. In fluids with metal nanoparticles, laser excitation leads to generate hot electrons which thermalized by electron phonon scattering. This phenomenon can help to increase thermal diffusivity of nanoparticles. The suspension of solid particles in liquid media enhances its influences thermal diffusivity [8]. The changes in the thermal diffusivity of the suspension mainly depend on shape and size of the suspended particles $[9,10]$. In addition, the nature of the surface, such as charge, its distribution, and the presence of surfactant plays an important role [11, 12]. The subject of heat transport through dilute suspensions of solid particles has received a renewed boost owing to the reports of enhancement in thermal conductivity of suspensions carrying nanoparticles [13-16].

The TL [17-19] is a type of photothermal effect, which a laser with Gaussian intensity profile is used as an excitation laser that induces the beam temperature distribution in a sample. The heat generated is strongest at the center of the beam, because the beam intensity is strongest at that point. This temperature gradient produces a refractive index gradient which behaves like a converging or diverging lens depending on whether the change rate of refractive index with respect to temperature, $\mathrm{d} n / \mathrm{d} T$, is positive or negative $[20,21]$. The first measurement of the TL effect was performed by Gordon et al. in 1965 using a simple single-beam apparatus [22].

The enhancement in the thermal diffusivity values of solvents, such as ethanol, water, ethylene glycol, and culture medium, by the presence of Au nanoparticles has been studied [23, 24]. For example, with $0.033-\mathrm{mmol} \mathrm{Ag}$ in $50 \mathrm{ml}$ of ethanol, water and ethylene glycol made the 
thermal diffusivity increased by $0.7,7.0$, and $8.4 \%$, respectively. It was also reported that with 4 vol. $\%$ of nanoparticles of $\mathrm{CuO}$ (23.6 nm diameter) in ethylene glycol made the thermal diffusivity increased by $22 \%$ [25]. This phenomenon could be due to the electrostatic interaction between cationic solutions and negatively charged metal nanoparticles.

We have previously investigated the influence of particle size on thermal diffusivity of Au nanoparticles prepared by $\gamma$ irradiation in PVA solution [26], but in present work, we studied the effect of concentration and particle size of $\mathrm{Au} / \mathrm{PVP}$ nanoparticles which prepared using $\gamma$-radiation on thermal diffusivity by using the TL spectrometric measurements.

\section{Material and method}

For preparing the Au/PVP nanoparticles samples, hydrogen tetrachloroaurate (III) hydrate, premion (metals basis), (HAuCl4.3H2O with $99.999 \%$ purity), 4-g PVP (MW 29,000, Aldrich), and 1-ml isopropanol were used for preparing Au nanoparticles samples. The PVP and isopropanol were used as a colloidal stabilizer and hydroxyl radical scavenger, respectively. The PVP solutions were made by dissolving PVP powder in 50-ml deionized water at room temperature. The solution was magnetically stirred for $2 \mathrm{~h}$ and was bubbled with nitrogen gas $(99.5 \%)$ to remove oxygen. $\gamma$-radiation $\left({ }^{60}\right.$ Co-rays) was used as an effective tool for polymerization process and reducing agent same as procedure reported in our previous paper [27]. Hydrogen tetrachloroaurate (III) hydrate, $\mathrm{HAuCl}_{4} \cdot 3 \mathrm{H}_{2} \mathrm{O}$, at different weights (i.e. 2, 5, 10, 15, 20 and $30 \mathrm{mg}$ ) was added into PVP solution to produce Au nanoparticles samples. The $\mathrm{Au}$ ions with different concentrations in PVP solution were then irradiated with $\gamma$-radiation at dose of $50 \mathrm{kGy}$ to produce various concentrations and nanoparticle sizes, and were labeled as $\mathrm{S} 1=1.17 \times 10^{-4} \mathrm{M}$; $\mathrm{S} 2=2.94 \times 10^{-4} \quad \mathrm{M} ; \quad \mathrm{S} 3=5.88 \times 10^{-4} \quad \mathrm{M} ;$ $\mathrm{S} 4=8.83 \times 10^{-4} \quad \mathrm{M} ; \quad \mathrm{S} 5=11.76 \times 10^{-4} \quad \mathrm{M} ; \quad$ and $\mathrm{S} 6=17.64 \times 10^{-4} \mathrm{M}$. In this process, $\gamma$-irradiation produces hydrated electrons that reduce the $\mathrm{Au}$ ions to $\mathrm{Au}$ atoms, and then, the aggregated $\mathrm{Au}$ atoms are distributed in the solution. The characteristic of Plasmon peak of $\mathrm{Au}$ nanoparticle inside the fluid was detected using UV visible spectrometer (Lambda 25-Perkin Elmer), and the size of silver nanoparticles was determined using nanophox machine (Sympatec $\mathrm{GmbH}$, D-38678).

The average diameter of Au nanoparticles was measured using the nanophox machine (Sympatec GmbH, D-38678) and listed in Table 1. The shape of the particles and their polydispersity of a few samples were determined via transmission electron microscopy (TEM) (Hitachi H-7100). We found that the particles distribution in the solution are in uniform shape.

Schematic diagram of the TL experimental setup is shown in Fig. 1. The measurements were carried out using a diode laser (532 $\mathrm{nm}$ at $60 \mathrm{~mW}$, DPSS) as an excitation source and a $\mathrm{He}-\mathrm{Ne}$ laser (632.8 nm, $0.5 \mathrm{~mW}, \mathrm{HRP} 050$ ) as a probe beam. The excitation light beam was modulated by a variable frequency chopper (SR540), and then, it was focused by a lens of $21-\mathrm{cm}$ focal length onto a liquid sample contained in a 5-mm length quartz cuvette positioned at its focal plane. The Rayleigh parameter was found to be $16 \mathrm{~mm}$ which is larger than thickness of samples $(5 \mathrm{~mm})$, and hereby, the Rayleigh condition is satisfied. The probe beam was focused by a lens of $5 \mathrm{~cm}$ focal length away from the cuvette and aligned at an angle smaller than $1.5^{\circ}$ with respect to the excitation beam. The transmitted beam was detected by a photodiode detector (PD). An optical bandpass filter is placed in front of an aperture to remove the stray light from excitation beam entering PD. The PD output was coupled to a digital storage oscilloscope (Tektronix TDS 210) to record the time evolution of TL signal. The LabView software was used to capture the TL data from oscilloscope and to normalize it with respect to TL signal at time $t=0$.

A mechanical chopper was used for limiting the exposure of the sample to the light; when the hole is opened, the sample is illuminated, and light sent a signal to initialize the data acquisition. The maximum intensity of the probe beam was centered on the photodiode detector, and then signals, as a function of time, were registered and recorded by a detector, oscilloscope, and sent to a PC, through a GPIB interface bus. The analysis data must be extracted throughout the one period of whole signal and then normalized. The experimental data are fitted using theoretical Eq. (1) with $\theta$ and characteristic thermal time constant $\left(t_{\mathrm{c}}\right)$ as adjustable parameters. Now, thermal diffusivity of the sample can be easily calculated from Eq. (1). This process was applied for all experimental data to obtain the thermal diffusivity of samples.

\section{Theory}

The TL signal is defined as the normalized change of the transmission of the probe beam, centered at the beam axis and located at a distance much larger than the pump Rayleigh parameter. Shen et al. in 1995 have derived as expression for the signal using a diffraction approximation for Gaussian beams, which is given by [28]: 
Table 1 Au nanoparticles samples in PVP polymer prepared at different concentrations and irradiated for dose of $50 \mathrm{kGy}$

\begin{tabular}{llllll}
\hline Nanofluid samples & $\begin{array}{l}\text { Concentration } \\
\left(\times 10^{-4} \mathrm{M}\right)\end{array}$ & $\begin{array}{l}\text { Average particle } \\
\text { size }(\mathrm{nm})\end{array}$ & $t_{\mathrm{c}}\left(\times 10^{-3} \mathrm{~s}\right)$ & $\theta$ & $D\left(\times 10^{-3} \mathrm{~cm}^{2} / \mathrm{s}\right)$ \\
\hline S1 & 1.17 & 10 & $9.43 \pm 0.24$ & $1.254 \pm 0.06$ & $1.23 \pm 0.04$ \\
S2 & 2.94 & 15 & $6.91 \pm 0.21$ & $1.121 \pm 0.02$ & $1.68 \pm 0.14$ \\
S3 & 5.88 & 19 & $5.95 \pm 0.22$ & $1.232 \pm 0.04$ & $1.95 \pm 0.07$ \\
S4 & 8.83 & 32 & $4.58 \pm 0.22$ & $1.152 \pm 0.05$ & $2.53 \pm 0.11$ \\
S5 & 11.76 & 45 & $4.01 \pm 0.19$ & $2.245 \pm 0.02$ & $2.89 \pm 0.13$ \\
S6 & 17.64 & 60 & $3.72 \pm 0.19$ & $2.321 \pm 0.03$ & $3.12 \pm 0.13$ \\
\hline
\end{tabular}

Fig. 1 Schematic diagram of TL experimental setup

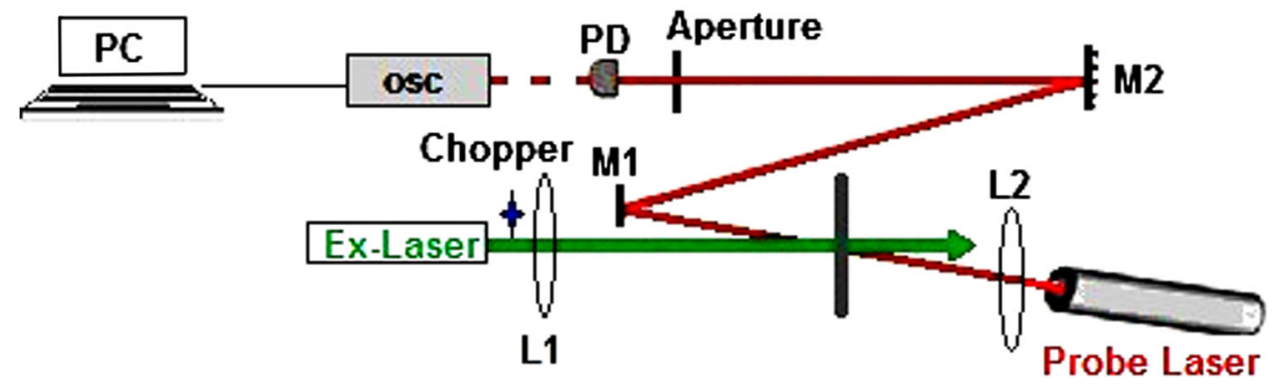

$I(z, t)=I_{0}\left(1-\theta \tan ^{-1}\left(\frac{2 \mathrm{mV}}{\left[(1+2 m)^{2}+V^{2}\right] \frac{t_{c}}{2 t}+1+2 m+V^{2}}\right)\right)^{2}$

where

$V=\frac{z_{1}}{z_{c}} ; m=\left(\frac{w_{p}}{w_{e}}\right)^{2} ; t_{c}=\frac{w_{e}^{2}}{4 D}, \theta=\frac{P_{e} \cdot \alpha \cdot l}{\kappa \lambda_{P}} \frac{\mathrm{d} s}{\mathrm{~d} T}$

in which $\lambda_{p}$ is the wavelength of the probe beam, D and $\kappa$ are the thermal diffusivity and thermal conductivity, $P_{e}$ is the excitation beam power, $\alpha$ is the absorption coefficient, and $l$ is the sample thickness. $z_{\mathrm{c}}=w_{\mathrm{o}}^{2} / \lambda$ is the confocal distance $(\mathrm{cm}), w_{\mathrm{O}}$ is the probe beam waist radius, $w_{\mathrm{p}}$ and $w_{\mathrm{e}}$ are the probe and the excitation beam radii at sample, respectively, $z_{1}$ is the distance of laser beam waist to sample, and $t_{\mathrm{c}}$ is the characteristic thermal time constant. In Eq. (1), $I(t)$ is the temporal dependence of the probe beam intensity at the detector, $I_{\mathrm{o}}$ is the initial value of, $I(t) . \mathrm{d} s / \mathrm{d} T$ is the temperature coefficient of the optical path length change, at the probe beam wavelength, which is written as [29]:

$\frac{\mathrm{d} s}{\mathrm{~d} T}=(n-1)(1+v) \alpha_{T}+\frac{\mathrm{d} n}{\mathrm{~d} T}$

where $\alpha_{\mathrm{T}}$ is the sample linear thermal expansion coefficient, $v$ is the Poisson ratio, and $\mathrm{d} n / \mathrm{d} T$ is the temperature coefficient of its refractive index.

\section{Results and discussion}

The absorption spectra of the Au nanoparticle prepared using $\gamma$-radiation is shown in Fig. 2. The measurements of absorption spectra were carried out at room temperature for visible wavelength, $380-800 \mathrm{~nm}$. As it can be seen, absorbance values increase with increasing the concentration of $\mathrm{Au}$ nanoparticles which indicated the total number of particles in the solution. Furthermore, the absorption peak shifted to lower energy or higher wavelengths with growth of nanoparticle sizes [30].

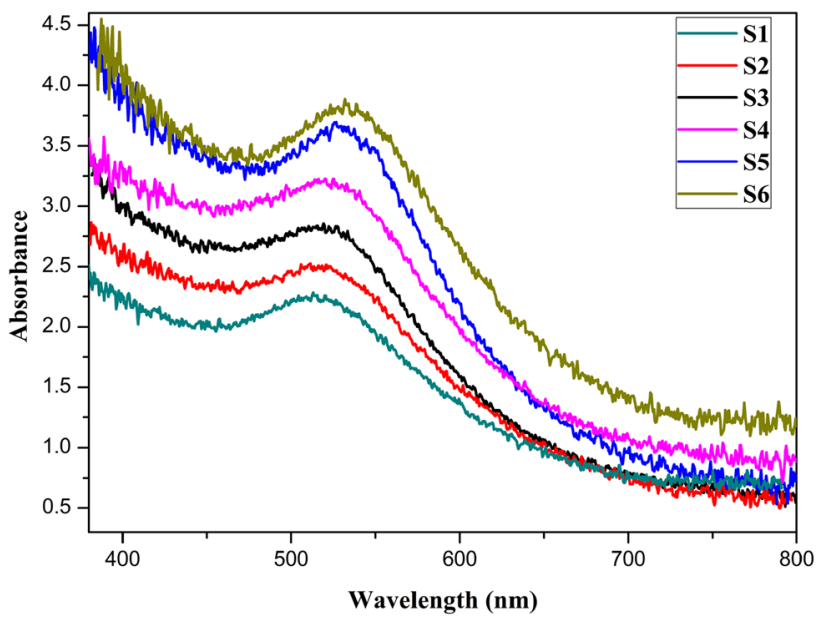

Fig. 2 Absorption spectra of the different concentration $\mathrm{Au}$ nanoparticles 


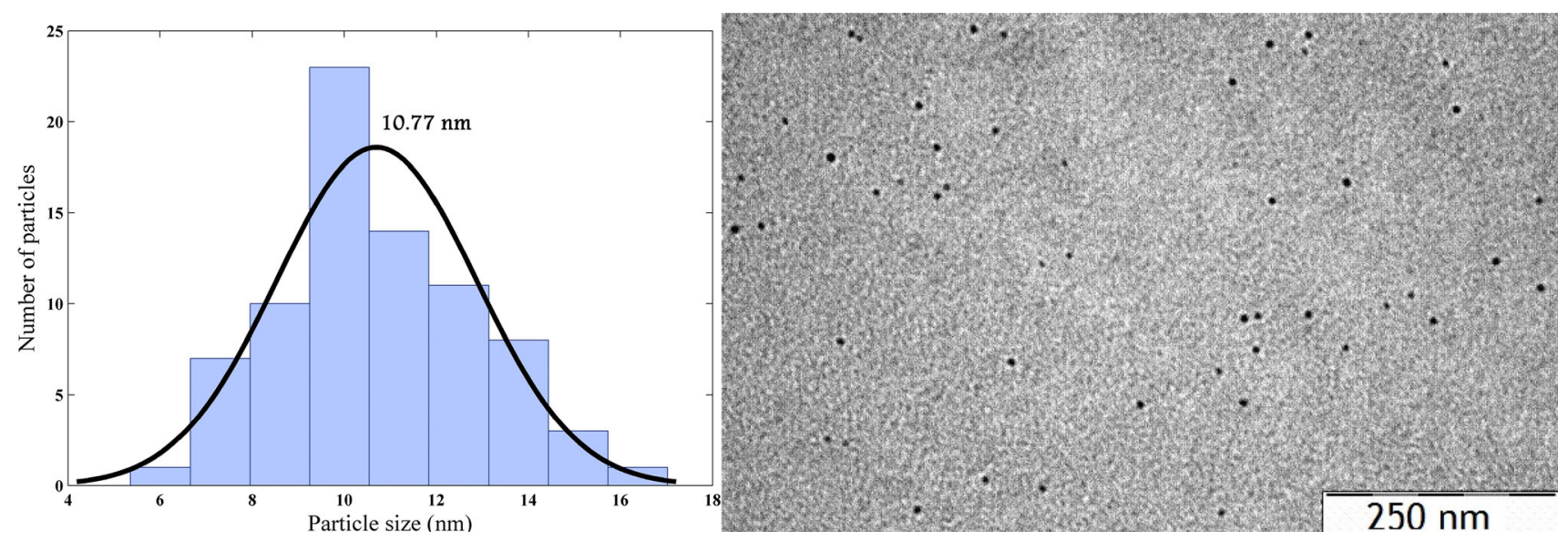

Fig. 3 TEM image and particles size histogram of Au particles with a nominal size of $10 \mathrm{~nm}$

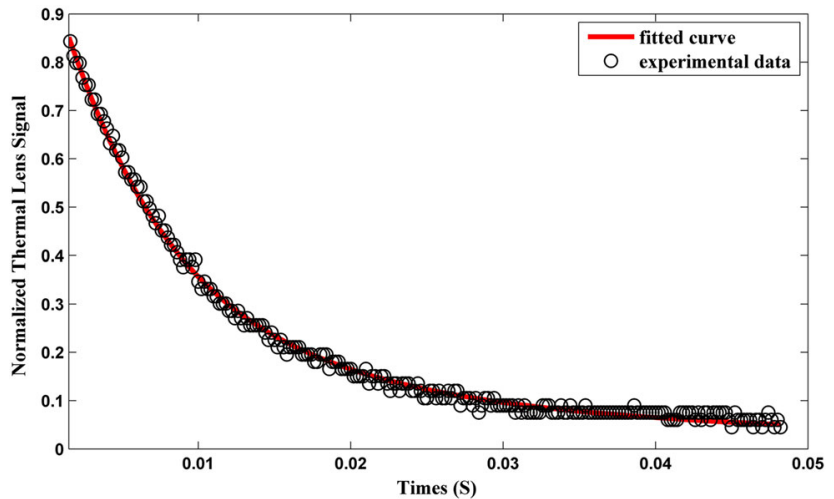

Fig. 4 Typical time evolution of the TL signal for Au nanoparticles (S1). Symbol (O) represents the experimental data and solid line is the best fitting line to Eq. (1)

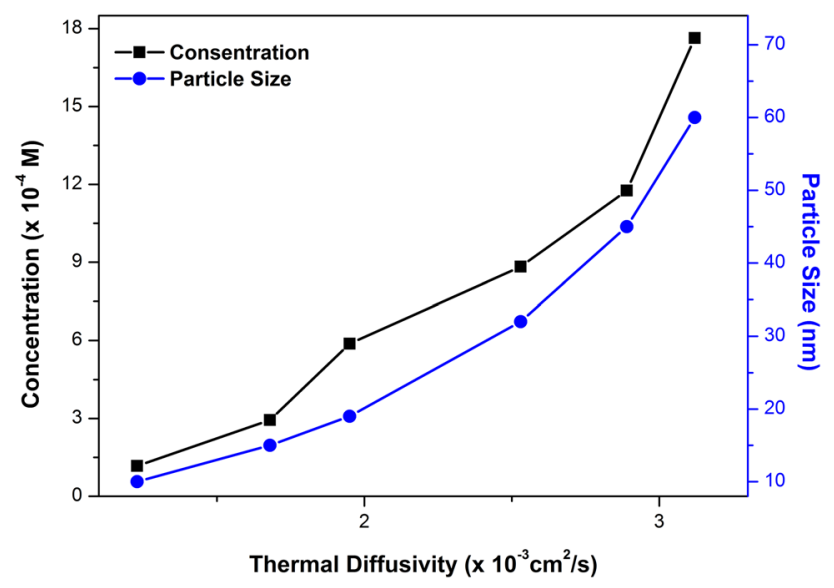

Fig. 5 Variations of thermal diffusivity of Au nanoparticles as a function of concentration and particle size

The particle size can be controlled by controlling the doses of $\gamma$-radiation, however, in the present work, the specimens were prepared at different concentration levels and radiated at $50 \mathrm{kGy}$. A typical TEM image of $\mathrm{Au}$ nanoparticles distributions with a nominal size of $10 \mathrm{~nm}$ and shape of these particles displayed in Fig. 3. Other samples of various concentrations or sizes of nanoparticles after irradiated at $50 \mathrm{kGy}$ can be seen in Table 1 .

Figure 4 shows a typical TL signal as a function of time for Au nanoparticles dispersed in PVP at concentration of $1.17 \times 10^{-4} \mathrm{M}$ and irradiated with dose of $50 \mathrm{kGy}$. The theoretical curves in Fig. 4 calculated using Eq. (1) agree very well with experimental data, which give the thermal diffusivity value for the sample as $1.23 \times 10^{-3} \mathrm{~cm}^{2} / \mathrm{s}$.

Thermal diffusivity value as a function of concentrations and nanoparticles sizes plotted in Fig. 5 which shows a nonlinear increasing with the increasing of concentration of nanoparticles and particles sizes. This enhancement can be due to the phonon scattering phenomena between the particles-liquid interfaces. Since, as the particle size becomes small, the phonon scattering transfers less energy to the surrounding liquid [31]. Moreover, the energy deposited into the phonon modes is subsequently transferred to the surrounding medium. In addition, when the particle concentration is increased, the optical absorption intensity is increased, as well as the thermal diffusivity of the surrounding medium. This interaction implies a higher nanoparticle concentration per volume unit in the solution [32].

\section{Conclusion}

We investigated thermal diffusivity treatment of $\mathrm{Au}$ nanoparticle at different concentrations and particle sizes. According to the experimental results with the increment of concentration and particle size of nanoparticle, the thermal diffusivity of samples increased. This increment in thermal diffusivity can be attributed to phonon scattering at interface of particles-liquid and contact between the nanoparticles and surrounded liquid. 
Open Access This article is distributed under the terms of the Creative Commons Attribution 4.0 International License (http://crea tivecommons.org/licenses/by/4.0/), which permits unrestricted use, distribution, and reproduction in any medium, provided you give appropriate credit to the original author(s) and the source, provide a link to the Creative Commons license, and indicate if changes were made.

\section{References}

1. Gittins, D.I., Bethell, D., Schiffrin, D.J., Nichols, R.J.: A nanometer scale electronic switch consisting of a metal cluster and redox-addressable groups. Nat. (London) 408, 67-69 (2000)

2. Ashkarran, A.A.: Synthesis and characterization of gold nanoparticles via submerged arc discharge based on a seed-mediated approach. J. Theo. Appl. Phys. 6, 14-16 (2012)

3. Neal, D.P.O., Hirsch, L.R., Halas, N.J., Payne, J.D., West, J.L.: Photo-thermal tumor ablation in mice using near infrared-absorbing nanoparticles. Cancer Lett. 209, 171-176 (2004)

4. Wen, D.S., Ding, W.: Natural convective heat transfer of suspensions of titanium dioxide nanoparticles (Nanofluids). IEEE Trans. Nanotechnol. 5, 220-227 (2006)

5. Jang, S.P., Choi, S.U.S.: Cooling performance of a microchannel heat sink with nanofluids. Appl. Therm. Eng. 26, 2457-2463 (2006)

6. Hamad-Schifferli, K., Schwartz, J.J., Santos, A.T., Zhang, S.G., Jacobson, J.M.: Remote electronic control of DNA hybridization through inductive coupling to an attached metal nanocrystal antenna. Nat. (London) 415, 152-155 (2002)

7. Loo, C., Lin, A., Hirsch, L., Lee, M.H., Barton, J., Halas, N., West, J., Drezek, R.: Nanoshell-enabled photonics-based imaging and therapy of cancer. Tech. Cancer Res. Treat. 3, 33-40 (2004)

8. Deen, W.M.: Analysis of transport phenomena. Oxford University Press, New York (1998)

9. Abdi, F., Siabi-Gerjan, A., Savaloni, H.: On the discrete dipole approximation investigation of the extinction spectra of Ag/glass nano-flower thin film with threefold symmetry. J. Theo. Appl. Phys. 6(4), 2-10 (2012)

10. Chopkar, M., Sudarshan, S., Das, P., Manna, I.: Effect of particle size on thermal conductivity of nanofluids. Metall. Mater. Trans. A 39, 1535 (2008)

11. Pilkington, G.A., Briscoe, W.H.: Nanofluids mediating surface forces. Adv. Coll. Interface Sci. 68, 179-182 (2012)

12. Gao, J.W., Zheng, R.T., Ohtani, H., Zhu, D.S., Chen, G.: Experimental investigation of heat conduction mechanisms in nanofluids clue on clustering. Nano Lett. 9, 4128 (2009)

13. Eastman, J.A., Phillpot, S.R., Choi, S.U.S., Keblinski, P.: Thermal transport in nanofluids. Annu. Rev. Mater. Res. 34, 219 (2004)

14. Fan, J., Wang, L.: Review of heat conduction in nanofluids. J. Heat Transfer 133, 040801 (2011)

15. Keblinski, P., Prasher, R., Eapen, J.: Thermal conductance of nanofluids: is the controversy over. J. Nanopart. Res. 10, 1089 (2008)
16. Kleinstreuer, C., Feng, Y.: Experimental and theoretical studies of nanofluids thermal conductivity enhancement: a review. Nanoscale Res. Lett. 6, 229 (2011)

17. Franko, M.: Thermal lens spectrometric detection in flow injection analysis and separation techniques. Appl. Spectrosc. Rev. 43, 358-388 (2008)

18. Kitamori, T., Tokeshi, M., Hibara, A., Sato, K.: Thermal lens microscopy and microchip chemistry. Anal. Chem. 76, 52A-60A (2004)

19. Cognet, A., Berciaud, S., Lasne, D., Lounis, B.: Photothermal methods for single nonlumimescent nano objects. Anal. Chem. 80, 2289-2294 (2008)

20. Bialkowski, S.: Photothermal spectroscopy methods for chemical analysis. Wiley, New York (1996)

21. Shen, J., Lowe, R.D., Snook, R.D.: Two-beam thermal lens spectrometer for. Ultra-trace Analysis. Chem. Phys. 18, 403-408 (1998)

22. Gordon, J.P., Leite, R.C.C., Moore, R.S., Porto, S.P.S., Whinnery, J.R.: Long-transient effects in lasers with inserted liquid samples. J. Appl. Phys. 36, 3-8 (1965)

23. Perez, J.L.J., Fuentes, R.G., Ramirez, J.F.S., Orea, A.C.: Study of gold nanoparticles effect on thermal diffusivity of nanofluids based on various solvents by using thermal lens spectroscopy. Eur. Phys. J. Spec. Top. 153, 159-161 (2008)

24. Perez, J.L.J., Fuentes, R.G., Alvarad, E.M., Gallegos, E.R., Orea, A.C., Cordova, J.T., Alvarez, J.G.M.: Enhancement of the thermal transport in a culture medium with Au nanoparticles. Appl. Surf. Sci. 255, 701-702 (2008)

25. Xue, Q., Xu, W.M.: A model of thermal conductivity of nanofluids with interfacial shells. Mater. Chem. Phys. 90, 298-301 (2005)

26. Shahriari, E., Yunus, W.M., Zamiri, R.: The effect of nanoparticle size on thermal diffusivity of gold nano-fluid measured using thermal lens technique. J. Eur. Opt. Soc. Rap. Public. 8, 13026 (2013)

27. Shahriari, E., Yunus, W.M., Saion, E.: Effect of particle size on nonlinear refractive index of au nano particle in PVA solution. Braz J. Phys. 40, 256-260 (2010)

28. Shen, J., Lowe, R.D., Snook, R.D.: Two-beam thermal lens spectrometer for ultra-trace analysis. Chem. Phys. 18, 403-408 (1998)

29. Andrade, A.A., Catunda, T.: Thermal lens determination of the temperature coefficient of optical path length in optical materials. Rev. Sci. Inst. 74, 877-880 (2003)

30. Naghavi, K., Saion, E., Yunus, W.M.: Influence of dose on particle size of colloidal silver nanoparticles synthesized by gamma radiation. Radiat. Phys. Chem. 79, 1203-1208 (2010)

31. Ge, Z.B., Cahill, D.G., Braun, P.V.: Thermal conductance of hydrophilic and hydrophobic interfaces. Phys. Rev. Lett. 96, 186101-186105 (2006)

32. Rotkin, S.V., Perebeinos, V., Petrov, A.G., Avouris, P.: An essential mechanism of heat dissipation in carbon nanotube electronics. Nano Lett. 9, 1850-1855 (2009) 